\title{
PRODUTOS QUÍMICOS E PRÉ-TRATAMENTOS EMPREGADOS NO PSM PARA CONTROLE DE INCRUSTAÇÃO: UMA REVISÃO
}

\author{
Danielle Silva Santos ${ }^{1}$; Edna dos Santos Almeida² \\ ${ }^{1}$ Senai Cimatec; Salvador/Bahia; daniellesantosegq@outlook.com \\ 2 Senai Cimatec; Salvador/Bahia; ednasa@fieb.org.br
}

\begin{abstract}
Resumo: O presente estudo tem como objetivo realizar uma revisão sistemática acerca da utilização de produtos químicos e pré-tratamentos para o controle de incrustação em processos de separação por membrana PSM. As buscas foram realizadas na base de dados Web of Science no período de 2008 a 2018, empregando palavras-chaves relacionadas ao objetivo. Os artigos foram classificados da seguinte forma: Uso de apenas um produto químico; Uso de mais de um produto químico; Uso de produtos químicos seguido de outro tipo de tratamento. Os pré-tratamentos combinado de produto químico com outro tipo de tratamento e uso de mais de um produto químico foram os mais citados nos artigos consultados e que apresentaram melhores resultados na redução de incrustação de membranas.
\end{abstract}

Palavras-Chave: incrustação de membrana; produtos químicos para tratamento de água/efluente; pré-tratamentos; separação por membranas.

\section{CHEMICAL PRODUCTS AND PRETRATMENTS USED IN MPS FOR THE FOULING CONTROL: A REVIEW}

\begin{abstract}
The present study aims to conduct a systematic review on the use of chemical products and pre-treatments for the fouling control in membrane separation processes MPS. The research was carried out in the Web of Science database from 2008 to 2018, using keywords related to the objective. The articles were classified as follows: Use of only one chemical products; Use of more than one chemical product; Use of chemicals products in conjunction with other treatment. The chemical products combination with another alternative and use of more than one chemical products were the most mentioned in the articles consulted and the one that performed the best results in the reduction of membrane incrustation.
\end{abstract}

Keywords: membrane fouling; chemicals products; Chemicals products for water/effluent treatment; pretreatments; membrane separation. 


\section{INTRODUÇÃO}

A partir do crescente interesse no tocante a preservação do meio ambiente aliado à cobrança pelo uso da água de maneira sustentável, que tem aumentado o uso de fontes e tratamentos alternativos para o abastecimento de água, os processos de separação por membranas (PSMs) vêm aumentando e se desenvolvendo muito nas últimas décadas, por possuírem uma capacidade de adequação as mais diversas aplicações, permitindo a reutilização das águas e, consequentemente, diminuindo o consumo de água de boa qualidade em processos que não necessitam de uma qualidade elevada, otimizando sua utilização nos processos industriais [1].

Os PSMs utilizam como barreira seletiva as membranas sintéticas e, através destas, é possível separar, concentrar e purificar substâncias. Estes processos são utilizados com o objetivo de separar da água substâncias e sólidos que têm pequenos diâmetros, além de moléculas e compostos iônicos, através da aplicação de algum tipo de força externa [2].

Diferentes PSMs estão disponíveis para a remoção de grande variedade de poluentes. Sua seleção é de acordo com a natureza do composto a ser removido. Os processos de microfiltração (MF) e ultrafiltração (UF) são comumente empregados para a eliminação da carga orgânica, na forma sólidos suspensos e macromoléculas, e podem ser utilizados tratamento principal ou como pré-tratamento ou visando a remoção de sais dissolvidos por nanofiltração (NF) e osmose reversa (OR), que também processos de separação por membrana [3].

As tecnologias de membrana apresentam um potencial de aplicação promissor com várias vantagens, dentre as quais se destacam a eficácia na remoção de poluentes, aumentando a qualidade do produto, automação no controle operacional, na manutenção e na substituição de equipamentos [4]. Entretanto, um dos grandes obstáculos enfrentados durante a maioria dos processos é o fenômeno da incrustação, que consiste na disposição ou acumulação de sólidos na superfície da membrana, causando bloqueio parcial ou total dos poros e podem ser decorrentes de formação de "bolo" e formação de camada de gel [1].

Segundo Lerch et al. [5], o entendimento majoritário sobre incrustações de membrana, elucida os seguintes aspectos negativos e, por conseguinte algumas desvantagens: redução significativa do fluxo do permeado, aumento dos custos operacionais e exigência de maior frequência de limpeza química. A limpeza pode eventualmente resultar em perda parcial da capacidade de filtração das membranas, consequentemente, diminuindo a vida útil das mesmas. 
Para mitigar o problema da incrustação é necessário um estágio de prétratamento ou emprego de produtos químicos, de modo que a maioria dos poluentes sejam removidos. Neste contexto, o principal objetivo deste trabalho é avaliar produtos químicos e pré-tratamentos usados para minimizar os efeitos negativos da formação de incrustações.

\section{METODOLOGIA}

Foram selecionados artigos publicados no período de 2008 a 2018 na base de dados de pesquisa Web of Science com enfoque nos estudos que avaliaram o uso de produto químico para redução de incrustação em PSMs no tratamento de efluentes/ água. As palavras-chaves utilizadas na pesquisa foram: "water reuse", "fouling", "microfiltration or ultrafiltration", "membrane near treatment", "wastewater or water near treatment", "coagulation or flocculation".

Os artigos de interesse potencial para este estudo foram aqueles aos quais apresentaram aspectos relevantes conforme os objetivos desta revisão. Os requisitos primordiais para seleção dos trabalhos foram balizados pela pertinência dos títulos e resumos buscados na base de dados Web of Science, a partir de então, foram selecionados para uma análise aprofundada e minuciosa no que tange a sua identificação com este estudo. Os estudos que não abordaram, de forma profundada, sobre incrustação em PSMs e que não avaliaram o efeito do uso produto químico como pré-tratamento para redução de incrustação foram excluídos.

Após o cumprimento das etapas anteriores, realizou-se uma leitura completa de cada estudo na tentativa de organizar as informações, com a elaboração de tabelas e gráficos através da ferramenta Microsoft Excel, de acordo com as seguintes nomenclaturas: (1) Referência; (2) Publicação; (3) Ano de publicação; (4) País; (5) Produtos Químicos. Em relação ao país, foi considerado a nacionalidade do primeiro autor citado na publicação.

Ademais, para oportunizar uma melhor avaliação dos artigos objeto deste estudo, estes foram agrupados segundo os tipos de métodos para controle incrustação em PSMs, com enfoque naqueles que apresentaram propostas de prétratamento aplicando produtos químicos. Sendo classificados da seguinte forma: Uso de apenas um produto químico; Uso de mais de um produto químico; Uso de produtos químicos seguido de outro tipo de pré-tratamento.

\section{RESULTADOS E DISCUSSÃO}

A pesquisa na base de dados revelou 39 (trinta e nove) artigos, sendo que 1 (um) artigo não se obteve acesso ao conteúdo, 14 (quatorze) artigos foram excluídos 
pela leitura dos títulos e resumos, e 5 (cinco) artigos eliminados pela leitura do texto integral. Por fim, o número final de artigos considerados para a fase de extração dos dados foi 19 (dezenove).

Os estudos de acordo com os anos de publicação podem ser observados em forma gráfica na Figura 1. O número de artigos que abordam o tema de utilização de produtos químicos como pré-tratamento para o controle de incrustação em PSM, não houve um crescimento linear ao longo do tempo. Do ano de 2008 (três artigos) a 2011(um artigo) houve uma queda significativa quanto ao número de publicações, porém em 2008, 2009 (três artigos) e 2015 (cinco artigos), foram os anos com um maior número de estudos publicados. Observa-se na Figura 2, em forma gráfica, que as Revistas Desalination e Desalination and Water Treatment são as que mais apresentaram artigos publicados referentes à temática com sete artigos e três artigos respectivamente.

Figura 1. Publicações no período de 2008 a 2018 na Web of Science

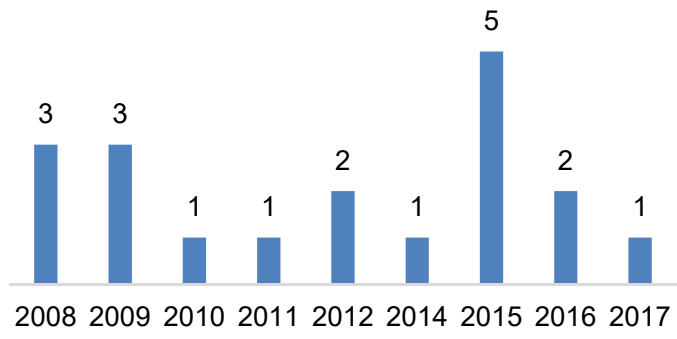

Figura 2. Revistas que publicaram no período de 2008 a 2018

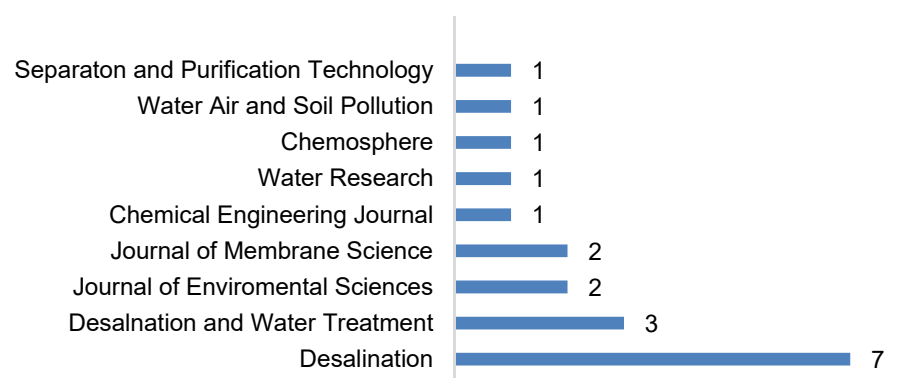

O tema estudado demonstrou ser de grande interesse para a comunidade científica, pois, a partir da realização do presente estudo, foi possível observar variedade significativa de países nos quais ocorreram publicações semelhantes. Destaca-se que, dos 19 estudos encontrados na revisão sistemática sobre prétratamentos com produtos químicos para redução de incrustação em membranas, apenas um deles é brasileiro, identificando uma lacuna na literatura nacional sobre o tema. Em contrapartida, os países que mais se destacaram em termos de número de publicação foram a Coreia com quatro artigos e a China com três artigos. Também foram encontrados estudos na Austrália, Canadá, Espanha, Croácia, Eslovênia, Índia, Estados Unidos, Taiwan e Tunísia.

Relativo aos produtos químicos que tiveram maior quantidade de citação na literatura revisada, os mais aplicados como pré-tratamentos foram os seguintes coagulantes inorgânicos classificados como: Sais de alumínio e Sais de ferro. Importante salientar, que estes coagulantes químicos, são os mais comercialmente utilizados, devido ao fato de terem baixo valor de mercado e fácil empregabilidade 
sendo que sua adequada dosagem permite economizar produtos químicos e tempo de agitação para floculação do efluente/água em tratamento. Além disso, por reduzirem notavelmente a matéria coloidal, devido à alteração da distribuição do tamanho das partículas da matéria orgânico, se tornam frações maiores, contribuindo, para a redução da taxa de incrustação. Corroborando assim para o fato de uma predileção por esses coagulantes, conforme apontado na Figura 3 [6].

Na Figura 4, está apresentada a classificação quanto ao uso de produtos químicos usados em processos de separação por membrana. Os métodos de prétratamentos classificados em uso de mais de um produto químico foram em maior quantidade, encontrados em $42 \%$ dos artigos analisados, seguido de produto químico combinado com outras alternativas de pré-tratamento com $32 \%$ Isto pode estar relacionado com o aumento da eficiência na redução de incrustação quando realizada combinação de dois ou mais produtos químicos, ou fazendo-se um sistema híbrido com procedimentos de filtração.

Figura 3. Produtos Químicos citados na revisão Figura 4. Classificação de métodos de pré-

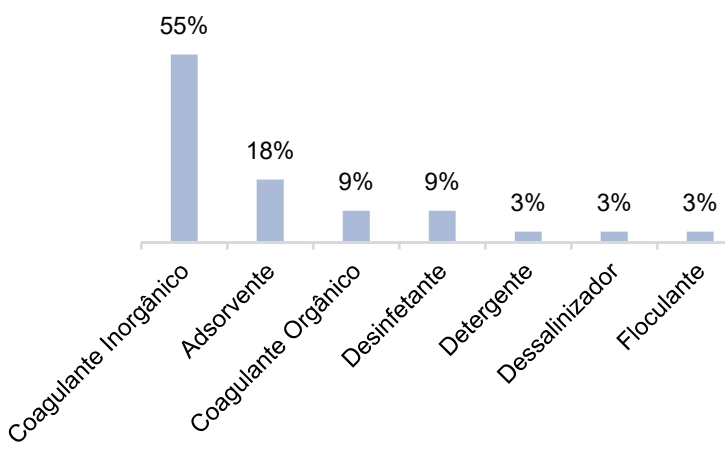
tratamentos que utilizaram produtos químicos em PSMs

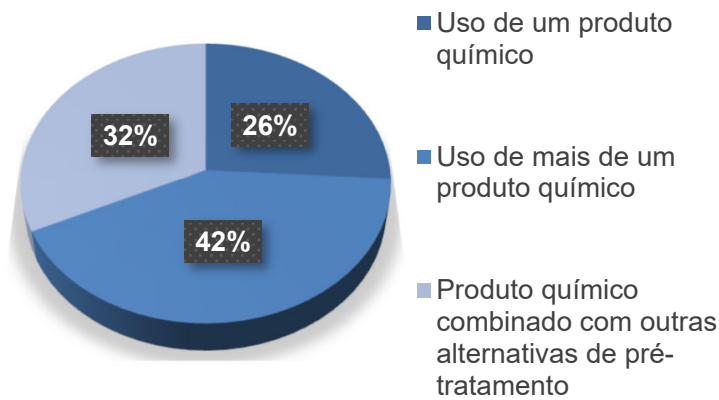

A Tabela 1 sumariza os principais resultados dos artigos selecionados nesta revisão.

Tabela 1: Principais resultados de alguns artigos selecionados nesta revisão

\begin{tabular}{|c|c|c|c|}
\hline PSM & PRÉ-TRATAMENTO & RESULTADOS & REFERÊNCIA \\
\hline $\begin{array}{l}\text { MF } \\
\text { NF }\end{array}$ & $\begin{array}{l}\text { Moringa Oleífera na } \\
\text { coagulação/floculação/sedimentação } \\
\text { (CFS) }\end{array}$ & $\begin{array}{l}\text { A taxa de incrustação para o tratamento CFS-MF- } \\
\text { NF foi de } 63 \% \text {, enquanto os tratamentos } \\
\text { restantes, CFS-MF e CFS-NF foi de } 89 \% \text { e } 91 \% \\
\text { respectivamente. }\end{array}$ & $\begin{array}{l}\text { Mateus, } \\
\text { G.A.P.; et al [7] }\end{array}$ \\
\hline NF & $\begin{array}{l}\text { Cloreto Férrico } \\
\text { Filtração em areia }\end{array}$ & $\begin{array}{l}\text { A incrustação foi aliviada substancialmente de } \\
74,4 \% \text { para } 12,3 \% \text { e houve redução do declínio } \\
\text { do fluxo das membranas de } 4,2 \text { a } 19,3 \text { vezes. }\end{array}$ & $\begin{array}{l}\text { Racar, M; et } \\
\text { al.[8] }\end{array}$ \\
\hline $\begin{array}{l}\text { MF } \\
\text { UF }\end{array}$ & $\begin{array}{l}\text { Alúmen } \\
\text { Amerfloc445 }\end{array}$ & $\begin{array}{l}\text { Os volumosos flocos de Amerfloc445 } \\
\text { proporcionaram melhores remoções de DQO e de } \\
\text { cor, no sistema de coagulação/UF. }\end{array}$ & $\begin{array}{l}\text { Masmoudi, G; } \\
\text { et al.[3] }\end{array}$ \\
\hline MF & EPI-DMA & $\begin{array}{l}\text { Aumento dez vezes maior no fluxo crítico, de } 45 \\
\mathrm{Lm}^{-2} \mathrm{~h}^{-1} \text { para } 450 \mathrm{Lm}^{-2} \mathrm{~h}^{-1} \text {, usando a coagulação } \\
\text { com dosagem de neutralização de carga. }\end{array}$ & $\begin{array}{l}\text { Shang, X.; et } \\
\text { al.[9] }\end{array}$ \\
\hline
\end{tabular}


MF Carvão ativado em pó (CAP)
Alúmen

\begin{tabular}{|c|l|}
\hline $\begin{array}{l}\text { OR } \\
\text { MF }\end{array}$ & Carvão Ativado Biológico (CAB) \\
\hline UF & $\begin{array}{l}\text { Carvão ativado em pó (CAP) } \\
\text { Cloreto Férrico }\end{array}$ \\
\hline UF & $\begin{array}{l}\text { Hidróxido de Sódio } \\
\text { Dodecil Sulfato de sódio (DSS) }\end{array}$ \\
\hline
\end{tabular}

\begin{tabular}{|c|l|}
\hline UF & Polieletrólito Catiônico \\
\hline MF & $\begin{array}{l}\text { Cloreto de Polialumínio } \\
\text { Ozônio }\end{array}$ \\
\hline
\end{tabular}

UF $\quad$ Carvão ativado em pó (CAP)

Alúmen

UF $\quad$ Carvão ativado em pó (CAP)

Alúmen

UF Cloreto de Polialumínio (PAC)

OR Filtração em areia (FA)

UF Cloreto Férrico

Ozônio

Sulfato Férrico

UF Alúmen

Carvão Ativado em pó (CAP)

MF Cloreto de Polialumínio (PAC) Ozônio

Alúmen

UF Cloreto Férrico

\begin{tabular}{|l|l|}
\hline & \\
\hline UF & Hipoclorito de sódio \\
OR & Cloreto Férrico
\end{tabular}

OR Cloreto Férrico

MF Alúmen

MF Cloreto Férrico

UF Resina de troca iônica
CAP combinado $\mathrm{cm}$ a coagulação do lodo de alúmen tem-se uma melhor remoção de partículas e matérias orgânicas.

O sistema híbrido $\mathrm{CAB}+\mathrm{MF}$, reduz efetivamente o carbono orgânico dissolvido biodegradável e o potencial de regeneração bacteriana, confirmando assim seu potencial para mitigar a incrustação biológica.

A combinação do CAP com o Cloreto Férrico foi mais favorável para a redução apreciável dos contaminantes emergentes.

Incrustação irreversível é mais acentuada no prétratamento com $\mathrm{NaOH}$ do que com o prétratamento de DSS.

O pré-tratamento resulta um fluxo maior devido à menor concentração dos coloides no efluente e a uma formação mais lenta da camada de bolo. Uma taxa de incrustação mais lenta é observada. Com a ozonização, mais de $90 \%$ da cor foi removida, e cerca de $82 \%$ de DQO foi reduzido, mesmo com uma dose baixa de ozônio ( $5 \mathrm{mg} / \mathrm{L})$. A pressão trasmembrana (PTM) da UF associada ao reuso de CAP combinado com alúmen foi relativamente estável durando 15 dias de operação, com PTM inicial 20,5 kPa e o PTM final de $21,5 \mathrm{kPa}$, o que indicou baixa incrustação da membrana.

Remoções de turbidez, DQO, UV254 foram 93,8, $37,3,41,1 \%$, em média, pelo pré-tratamento com o reuso de CAP combinado com alúmen.

Mais de $99 \%$ da turbidez e partículas podem ser removidos utilizando UF como pré-tratamento após a coagulação/floculação/FA.

O declínio do fluxo da membrana do UF diminuiu muito após a ozonização juntamente com a coagulação.

Ao contrário do pré-tratamento com coagulantes, a adsorção de carbono não reduziu a Rc (resistência formada pela camada depositada sobre a superfície da membrana), que é principal responsável pela incrustação da membrana.

Utilizando $1 \mathrm{mg} / \mathrm{L}$ de PAC e $4 \mathrm{mg} / \mathrm{L}$ de ozônio como pré-tratamento, demonstrou que, durante um período de 4 semanas, pouca ou nenhuma incrustação ocorreu e uma PTM estável foi mantida em 3 psi.

MF utilizando como pré-tratamento coagulação com alúmen ou cloreto férrico resultou em taxas de recuperação de fluxo variando de $88 \%$ a $94 \%$ em comparação com $61 \%$ para a água não tratada, reduzindo a incrustração irreversível da membrana.

Coagulação foi encontrada ser a técnica preferida à cloração para UF. $\mathrm{FeCl}_{3}$ reduziu o declínio do fluxo de UF em $43 \%$ após os três dias de operação. Isso ocorreu principalmente devido à diminuição de $38 \%$ da carga orgânica.

Combinando coagulação de alúmen com tratamento de troca aniônica proporcionou uma redução significativa de matéria com alto fluxo e incrustações pouco irreversíveis.
Gong, $\mathrm{H}$.; et al. [10]

Pramanik, B. K.; et al. [11]

Acero, J. L.; et al.[12]

Singh, S. K.; et al. [13]

Simonic, M.; et al.[14]

Park, C.; et al.[15]

Qi, Lu; et al.[16]

Qi, Lu; et al.[17]

Yang, B.M.; et al. [18]

Jeong, K.; et al [19]

Baek, S.; Chang, I.

[20]

Lehman, S. G.; Liu, L. [21]

Citulski, J.; et al.[6]

Friedler, E.; e tal. [22]

Fan, L.; et al. [23] 


\section{CONCLUSÃO}

Este estudo apresentou uma revisão sistemática da literatura acerca da avaliação de PSMs para tratamento de efluente a partir da utilização de produtos químicos como pré-tratamento a fim de reduzir os efeitos negativos da formação de incrustações, tendo sido selecionados 19 artigos. Apenas um dos artigos da revisão é brasileiro, identificando uma lacuna na pesquisa nacional sobre o tema. Em contrapartida, os países que mais se destacaram em termos de número de publicação foi a Coreia e a China. As Revistas Desalination e Desalination and Water Treatment são as que mais apresentaram artigos publicados em função da temática de publicação destas revistas.

A partir da realização desta revisão, foi possível verificar que os coagulantes inorgânicos tiveram um alto percentual de citação com relação aos demais. Os estudos que avaliaram o uso de produto químico como outra alternativa de prétratamento e uso de mais de um produto químico, foram em maior número do que nos artigos que apenas utilizaram um do produto químico.

Verificou-se que o uso de mais de um produto químico, resultou em sua maioria, a associação de um coagulante e um adsorvente. Este pré-tratamento retarda o aumento da resistência à filtração reduzindo a formação de incrustação, ao mesmo tempo em que reduz a concentração de matéria orgânica. Na maioria dos estudos, realizaram-se pré-tratamentos que associaram produtos químicos com membranas. Importante destacar que, em grande parte dos estudos analisados o uso de produtos químicos em pré-tratamentos para controle de incrustações apontaram bons resultados, proporcionando altas eficiências de remoção de matéria orgânica, cor e turbidez e consequentemente reduzindo incrustações em PSMs.

\section{REFERÊNCIAS}

${ }^{1}$ ALVES, T.L. Estudo da formação de incrustações inorgânicas em membranas de nanofiltração utilizadas em processos de dessulfatação. 2006. 18 f. Tese (Doutorado em Ciência dos Materiais e Metalurgia) - Pontifícia Universidade Católica do Rio de Janeiro, Rio de Janeiro, 2006.

${ }^{2}$ GALVÃO, D. F.; GOMES, E. R. Dos S. Os processos de separação por membranas e sua utilização no tratamento de efluentes industriais da indústria de laticínios: revisão bibliográfica. Revista do Instituto de Laticínios Cândido Torres, Medianeira, v. 70, n. 6, p. 349-360, dez. 2015. 
${ }^{3}$ MASMOUDI, G.; ELLOUZE, E; BEM, A. R. Hybrid coagulation/membrane process treatment applied to the treatment of industrial dyeing effluent. Desalination and Water Treatment, v. 57, Ed. 15, p. 6781-6791,2016.

${ }^{4}$ BRACEIRO, A.P.S. Utilização de Tecnologia de Separação por Membranas na Reabilitação de Sistemas de Tratamento de Água. 142 f. Dissertação (Mestrado em Engenharia do Ambiente) - Faculdade de Ciências e Tecnologia, Universidade Nova de Lisboa, Lisboa, 2014.

${ }^{5}$ LERCH, A.; SIEDRATH, N.; BERG, P.; HEIJNEN, M.; GITIS, V.; UHL, W. Fouling minimized reclamation of secondary effluents with reverse osmosis (ReSeRO). Desalination and Water Treatment, v. 42, Ed. 1-3, p.181-188, 2012.

${ }^{6} \mathrm{CITULSKI}, \mathrm{J}$; FARAHBAKHSH, H.; KENT, F. The impact of in-line coagulant addition on fouling potential of secondary effluent at a pilot-scale immersed ultrafiltration plant. Journal of Membrane Science, p. 311-318,2008.

${ }^{7}$ MATEUS, G. A. P.; FORMENTINI-SCHMITT, D. M.; NISHI, L.; FAGUNDES-KLEN, M. R.; GOMES, R. G.; BERGAMASCO, R. Coagulation/Flocculation with Moringa oleifera and Membrane Filtration for Dairy Wasterwater Treatment. Water air and soil pollution, v. 228, Ed. 9, p. 228-342, 2017.

${ }^{8}$ RACAR, M.; DOLAR, D.; SPEHAR, A. Optimization of coagulation with ferric chloride as a pretreatment for fouling reduction during nanofiltration of rendering plant secondary effluent. Chemosphere, v. 181 p.485-491,2017.

${ }^{9}$ SHANG, XIA; KIM, HYUN-CHUL; HUANG, JIN-HUI; et al. Coagulation strategies to decrease fouling and increase critical flux and contaminant removal in microfiltration of laundry wastewater. Separation and Purification Technology, p. 44-50, 2015.

${ }^{10} \mathrm{GONG}$, HUI; JIN, ZHENGYU; WANG, XIAN; et al. Membrane fouling controlled by coagulation/adsorption during direct sewage membrane filtration (DSMF) for organic matter concentration. Journal of Environmental Sciences, p.1-7,2015.

${ }^{11}$ PRAMANIK, B. K.; et al. Assessment of biological activated carbon treatment to control membrane fouling in reverse osmosis of secondary effluent for reuse in irrigation. Desalination, p. 90-95,2015.

${ }^{12} A C E R O$, J. L; BENITEZ, F. J; REAL, F. J; TEVA. Coupling of adsorption, coagulation, and ultrafiltration processes for the removal of emerging contaminants in a secondary effluent. Chemical Engineering Journal, p. 1-8, 2012. 
${ }^{13}$ SINGH, S. K.; KRAEMER, M.; TRE'BOUET, D. Studies on treatment of a thermomechanical process effluent from paper industry using ultrafiltration for water reuse. Desalination and Water Treatment, p. 208-217, 2012.

${ }^{14}$ SIMONIC, M.; LOBNIK, A. The efficiency of a hybrid flocculation/UF process for a real dye-house. Desalination, p. 219-224,2011.

${ }^{15}$ PARK, C.; HONG, S.; CHUNG, T. H. Performance evaluation of pretreatment processes in integrated membrane system for wastewater reuse. Desalination, $p$. 673-676, 2010.

${ }^{16}$ QI, L.; LIANG, H.; WANG, Y.; LIB, G. Integration of immersed membrane ultrafiltration with the reuse of PAC and alum sludge (RPAS) process for drinking water treatment. Desalination, p. 440-444, 2009.

${ }^{17}$ QI, L.; LIUA, G; ZHENGA, X.; LIB, G. Reuse of PAC and alum sludge (RPAS) process: pretreatment to reduce membrane fouling. Desalination and Water Treatment, p. 2421-2428, 2015.

${ }^{18 Y A N G, ~ B . M . ; ~ H U A N G ~ C . J . ; ~ L A I ~ W . L . ; ~ C H A N G, ~ C . C . ; ~ K A O, ~ C . M . ~ D e v e l o p m e n t ~ o f ~ a ~}$ three-stage system for the treatment and reclamation of wastewater containing nanoscale particles. Desalination, p. 182-190, 2012.

${ }^{19}$ JEONG, K.; LEE, D.; KIM, D.; KO, S. Effects of ozonation and coagulation on effluent organic matter characteristics and ultrafiltration membrane fouling. Journal of Environmental Sciences, p.1325-1331, 2014.

${ }^{20}$ BAEK, S; CHANG, I. Pretreatments to control membrane fouling in membrane filtration of secondary effluents. Desalination, p. 153-163, 2009.

${ }^{21}$ LEHMAN, S. G; LIU, L. Application of ceramic membranes with pre-ozonation for treatment of secondary wastewater effluent. Water research, p. 2020-2028, 2009.

${ }^{22}$ FRIEDLER, E.; KATZ, I.; DOSORETZ, C. G. Chlorination and coagulation as pretreatments for greywater desalination. Desalination, p. 38-49, 2008.

${ }^{23}$ FAN L.; NGUYEN T.; RODDICK F. A.; HARRIS, J. L. Low-pressure membrane filtration of secondary effluent in water reuse: Pre-treatment for fouling reduction. Journal of Membrane Science, p. 135-142, 2008. 